\title{
Ratchets driven by harmonic and white noise
}

\author{
R. Bartussek ${ }^{a, *}$, P. Hänggi ${ }^{a}$, B. Lindner ${ }^{b}$, L. Schimansky-Geier ${ }^{b}$ \\ ${ }^{a}$ Institut für Physik, Universität Augsburg, Mernminger Strasse 6, D-86135 Augsburg, Germany \\ ${ }^{\mathrm{b}}$ Institut fïr Physik, Humboldt-Universität zu Berlin, Invalidenstr. 110, D-10115 Berlin, Germany
}

\begin{abstract}
The synthesis of overdamped Brownian motion in a periodic potential which lacks reflection symmetry (ratchet device) with unbiased nonequilibrium forces $\epsilon(t)$ yields an ordered, directed particle current. In this work, $\epsilon(t)$ is made up of a noisy oscillator dynamics (harmonic noise). Such noise is able to account for inertial dynamic features; as a consequence we observe multiple current reversals upon varying the noise correlation time of the colored noise $\epsilon(t)$. The precise form of the particle current is determined by use of matrix-continued-fraction methods. As a function of noise color the current depicts a characteristic bell-shaped behavior. The precise numerical results concur with both, limiting analytical exact results and with an approximation scheme (unified colored noise approximation).
\end{abstract}

Keywords: Fluctuation phenomena; Brownian motion; Colored noise; Ratchet dynamics

\section{Introduction}

One of the hallmarks of equilibrium statistical mechanics is that, apart from ring currents (such as persistent currents), directed currents do not occur. It thus appears at first sight paradoxical that such directed currents do occur in periodic structures without the application of external bias forces or thermal gradients, or alike, cf. the recent reviews in [1]. Such devices recently have attracted interest [1-10] in view of potential biological transport applications and novel mass separation techniques [10]. These systems are termed ratchets: The characteristic features for such devices are (i) a spatially periodic structure that lacks reflection-symmetry (saw-tooth like structure) and (ii) the action of an additive, temporally varying force $\epsilon(t)$ of zero average. Usually these temporal forces are assumed to be symmetric. Otherwise, a current emerges even in presence of spatial reflection symmetry [6]. The correlated nature of $\epsilon(t)$, whose autocorrelation is not related - via detailed balance - to the dissipative mechanism, brings the ratchet system away from a thermal equilibrium system. The source of asymmetry, either for the ratchet structure or the temporal perturbation $\epsilon(t)$, is essential: Symmetric nonequilibrium forcing $\epsilon(t)$ in a symmetric periodic potential results in a zero net current.

In the following, we shall focus on an overdamped 'Brownian Rectifier' that is modeled by a periodic ratchet potential $V(x)=V(x+1)$ of period 1 . This rectifier is exposed simultaneously to thermal Gaussian noise $\xi_{1}(t)$ of

\footnotetext{
* Corresponding author.
} 
strength 2D and correlation $\left\langle\xi_{1}(t) \xi_{1}(s)\right\rangle=\delta(t-s)$, and unbiased, colored Gaussian fluctuations $\epsilon(t)$. This defines a ratchet dynamics that - with all variables chosen in dimensionless form, for the scaling of ratchet parameters see Appendix $\mathrm{A}$ - has the form of a Langevin equation with $t w o$ additive noise sources, i.e.,

$$
\dot{x}=-\frac{\partial V(x)}{\partial x}+\epsilon(t)+\sqrt{2 D} \xi_{1}(t) .
$$

The two random sources are assumed to be independent. Eq. (1) describes a correlation ratchet [4-7,9]. In this work we use for $\epsilon(t)$ a noisy oscillator dynamics, termed harmonic noise [11]. By doing so, we implicitly mimic a source of inertia [8] via the coupling of the $x$-dynamics to the inertial dynamics inherent in harmonic noise $\epsilon(t)$, see again in Appendix A. Next we investigate the statistical properties of this two-dimensional Gauss-Markov process.

\section{Harmonic noise}

\subsection{Definition and properties}

This harmonic noise process $\epsilon(t)$ is defined through the stochastic differential equations [11]

$$
\dot{\epsilon}=u, \quad \ddot{\epsilon}=\dot{u}=-\Gamma u-\Omega_{0}^{2} \epsilon+\Omega_{0}^{2} \sqrt{2 Q} \xi_{2}(t),
$$

where $\xi_{2}(t)$ is Gaussian white noise as $\xi_{1}(t)$, and independent of $\xi_{1}(t)$. Eq. (2) yields a Gaussian process with zero average, $\langle\epsilon(t)\rangle=0$, and autocorrelation

$$
\langle\epsilon(t) \epsilon(0)\rangle=\frac{Q \Omega_{0}^{2}}{\Gamma} \exp \left(-\frac{\Gamma}{2}|t|\right)\left(\cos \Omega_{1} t+\frac{\Gamma}{2 \Omega_{1}} \sin \Omega_{1}|t|\right),
$$

when $\Omega_{1}^{2}:=\Omega_{0}^{2}-\Gamma^{2} / 4 \geq 0$. Likewise, for $\Gamma^{2} \geq 4 \Omega_{0}^{2}$, we find with $\bar{\Omega}_{1}^{2}:=\Gamma^{2} / 4-\Omega_{0}^{2}$,

$$
\langle\epsilon(t) \epsilon(0)\rangle=\frac{Q \Omega_{0}^{2}}{\Gamma} \exp \left(-\frac{\Gamma}{2}|t|\right)\left(\cosh \bar{\Omega}_{1} t+\frac{\Gamma}{2 \bar{\Omega}_{1}} \sinh \bar{\Omega}_{1}|t|\right) .
$$

As a consequence, its spectral density can be evaluated to read

$$
S_{\epsilon \epsilon}(\omega):=\int \mathrm{d} t\langle\epsilon(t) \epsilon(0)\rangle \mathrm{e}^{\mathrm{i} \omega t}=\frac{2 Q \Omega_{0}^{4}}{\Gamma^{2} \omega^{2}+\left(\Omega_{0}^{2}-\omega^{2}\right)^{2}} .
$$

It exhibits two peaks for $\Gamma^{2}<2 \Omega_{0}^{2}$ (or $\tau<2 / \Gamma$, see Eq. (6) below) with a local minimum at $\omega=0$. With $\Gamma^{2} \geq 4 \Omega_{0}^{2}$, the correlation function is semipositive valued for all times. The correlation time for harmonic noise $\tau$ $:=\int_{0}^{\infty} \mathrm{d} t|\langle\epsilon(t) \epsilon(0)\rangle| /\left\langle\epsilon^{2}\right\rangle$ can be analytically evaluated to yield

$$
\tau=\Gamma / \Omega_{0}^{2} \text {. }
$$

\subsection{Limiting behavior of harmonic noise}

When $\Gamma \rightarrow \infty, \Omega_{0}^{2} \rightarrow \infty$, and $\Gamma / \Omega_{0}^{2}=$ const $=\tau$, harmonic noise approaches Ornstein-Uhlenbeck noise of intensity $Q$ and correlation time $\tau$. Consequently, if in addition $\tau \rightarrow 0, \epsilon(t)$ approaches white noise of intensity $Q$.

The adiabatic limit occurs for $\Gamma \rightarrow 0$ with $\tau$ held fixed: With $\Omega_{0}^{2}=\Gamma / \tau$, and thus $\Omega_{0}^{2} \gg \Gamma^{2}$, we obtain $\langle\epsilon(t) \epsilon(0)\rangle=(Q / \tau) \cos \Omega_{0} t$. Thus, $\epsilon(t)$ takes on a Gaussian distribution of quasistatic values with $\left\langle\epsilon^{2}\right\rangle=Q / \tau$. 
A third limit of harmonic noise corresponds to a limiting deterministic harmonic oscillator. This limit follows from harmonic noise when $\Gamma \rightarrow 0, Q \rightarrow 0, Q / \Gamma=C$ finite, and $\Omega_{0}$ is held fixed. This procedure yields $\langle\epsilon(t) \epsilon(0)\rangle \rightarrow\left(Q \Omega_{0}^{2} / \Gamma\right) \cos \Omega_{0} t$. The amplitude $A$ of the harmonic oscillator dynamics can correspondingly be identified as $A^{2} \equiv 2 Q \Omega_{0}^{2} / \Gamma=2 \Omega_{0}^{2} C$.

\section{Numerical solution with matrix-continued-fractions}

The starting point for the numerical investigation of the ratchet system (1), (2) is the corresponding Fokker-Planck equation for the joint probability density $W(x, \epsilon, u ; t)$,

$$
\frac{\partial W(x, \epsilon, u ; t)}{\partial t}=\left[\frac{\partial}{\partial x}\left(\frac{\partial V}{\partial x}\right)-\epsilon \frac{\partial}{\partial x}-u \frac{\partial}{\partial \epsilon}+\frac{\partial}{\partial u}\left(\Gamma u+\Omega_{0}^{2} \epsilon\right)+D \frac{\partial^{2}}{\partial x^{2}}+Q \Omega_{0}^{4} \frac{\partial^{2}}{\partial u^{2}}\right] W(x, \epsilon, u ; t) .
$$

In order to calculate the asymptotic probability current $J=\langle\dot{x}\rangle$ along the ratchet potential, where $\langle\dot{x}\rangle$ denotes the particle current, only the $x$-periodic stationary distribution $W_{\mathrm{st}}(x, \epsilon, u):=W(x, \epsilon, u ; t \rightarrow \infty)=W_{\mathrm{st}}(x+1, \epsilon, u)$ has to be evaluated. We normalize $W_{\mathrm{st}}(x, \epsilon, u)$ to unity within one spatial period, i.e., $\int_{0}^{1} \mathrm{~d} x \int_{-\infty}^{\infty} \mathrm{d} \epsilon \int_{-\infty}^{\infty} \mathrm{d} u W_{\mathrm{st}}(x, \epsilon, u)$ $\equiv 1$.

To apply the method of matrix-continued-fractions (MCF) to the calculation of $W_{\mathrm{st}}(x, \epsilon, u)$, we expand the potential $V(x)$ and the probability distribution into Fourier series in $x$, and into Hermite functions for the $u$ - and $\epsilon$-dependence [9,12]. The probability current in $x$-direction is given from $(7)$ as $J_{x}(x, \epsilon, u):=[-\partial V(x) / \partial x+\epsilon-$ $D \partial / \partial x] W_{\mathrm{st}}(x, \epsilon, u)$ and the total fluctuation-induced current $J$ is obtained as

$$
J:=\int_{0}^{1} \mathrm{~d} x \int_{-\infty}^{\infty} \mathrm{d} \epsilon \int_{-\infty}^{\infty} \mathrm{d} u J_{x}(x, \epsilon, u) .
$$

For concrete calculations, we use the same examples of ratchet potentials $V(x)$ as in [7], namely

$$
\begin{aligned}
& V_{2}(x)=-[\sin (2 \pi x)+0.25 \sin (4 \pi x)] /(2 \pi), \\
& V_{3}(x)=-\{\sin (2 \pi x)+0.2 \sin [4 \pi(x-0.45)]+0.1 \sin [6 \pi(x-0.45)]\} /(2 \pi),
\end{aligned}
$$

both with a smaller average force in 'forward' direction (see Fig. 1).

For the potential $V_{3}$, it was shown in [7], that the current for Ornstein-Uhlenbeck noise exhibits a change of its direction (current reversal) upon variation of $\tau$. This result for Ornstein-Uhlenbeck noise is shown in Fig. 2 by the solid line. The dashed, dotted and dashed-dotted lines present MCF-results for harmonic noise with $\Gamma$ finite. $A$ second current reversal appears generically at small $\tau$-values. These two current reversals occur in a regime where the corresponding spectrum $S_{\epsilon \epsilon}(\omega)$ of harmonic noise is doubly peaked at $\omega= \pm \sqrt{\Omega_{0}^{2}-\Gamma^{2} / 2}$. Within our variation

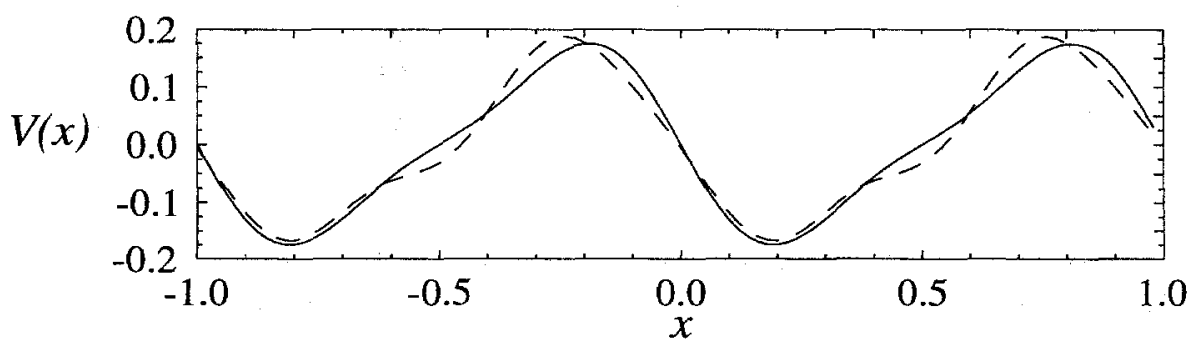

Fig. 1. The two types of ratchet potentials given in Eqs. (9) and (10) are shown as solid and dashed lines for $V_{2}(x)$ and $V_{3}(x)$, respectively. 


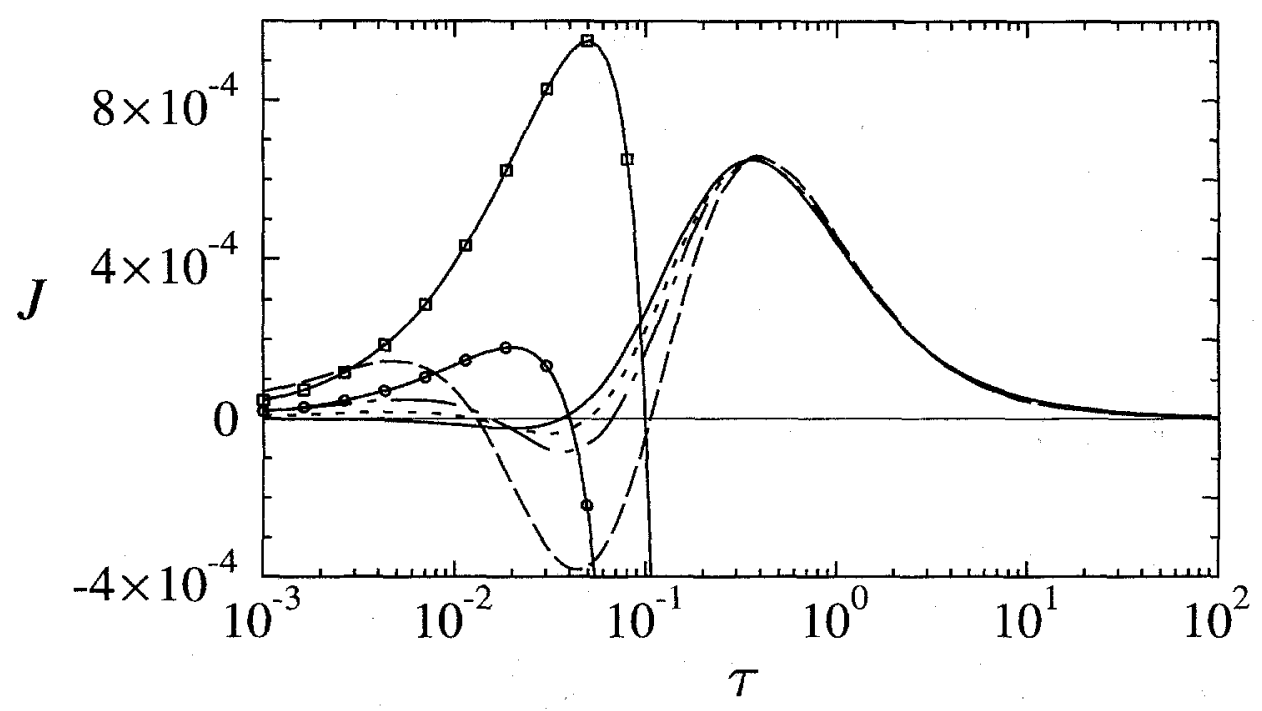

Fig. 2. The noise induced probability current $J$ (which here is equivalent to the particle current $\langle\dot{x}\rangle$ ) is drawn versus the noise correlation time $\tau$ for the ratchet potential $V(x)=V_{3}(x)$. The dimensionless noise parameters, cf. Eqs. (1) and (2), are chosen as $D=0.05$, $Q=0.025$, and different values for the damping: $\Gamma=20$ (dashed), 50 (dashed-dotted), 100 (dotted), and $\Gamma \rightarrow \infty$ (solid). The latter corresponds to Ornstein-Uhlenbeck noise with correlation time $\tau=\Gamma / \Omega_{0}^{2}$, see Section 2.2 . Two current reversals occur only when the parameter regime covers the case of a doubly peaked spectral function $S_{\epsilon \epsilon}(\omega)$, cf. Eq. (5). The theoretical prediction of [5] is drawn for $\Gamma=20$ (circles) and for $\Gamma=50$ (squares), note the description in the text.

of the three parameters characterizing harmonic noise in Eq. (2) we did not detect triple and higher order subsequent reversals of current. In this context, we remark that the inertial dynamics with deterministic rocking (possessing with particle mass, frequency, and strength of rocking also three independent parameters) is even richer [8]: When driven in the regime of chaotic transport trajectories the current exhibits (infinitely) many reversals. Flashing ratchets which are driven by a cyclic 3-state noise [13] also are able to exhibit two subsequent reversals of current. This effect could be of use for the selection of a certain species of particles, since the correlation time $\tau$ depends on the properties of the particle such as the friction coefficient, cf. Appendix A. Thus, particles whose friction values vary within a characteristic 'window' can be selected. The remaining lines in Fig. 2 give the theoretical predictions of [5]. After correcting misprints in the prefactor $W_{k}$, i.e., instead of using $W_{k}=\pi D / F(0) \sqrt{U^{\prime \prime}\left(x_{0}\right)\left|U^{\prime \prime}\left(x_{+}\right)\right|}$as given in [5] we use the Smouchowski result $W_{k}=\sqrt{U^{\prime \prime}\left(x_{0}\right)\left|U^{\prime \prime}\left(x_{+}\right)\right|} / 2 \pi$. The theory of [5] predicts a current reversal if the quantity $F^{\prime \prime}(\omega=0):=Q\left[\Omega_{0}(Q+D)\right]^{-2} \Gamma(\tau-2 / \Gamma) \max _{\omega} S_{\epsilon \epsilon}(\omega)$ is allowed to undergo a change of sign. In Fig. 2, the theory of [5] predicts a single (and not two) reversal at $\tau=0.1$ (for $\Gamma=20$ ), and $\tau=0.04$ (for $\Gamma=50$ ), respectively. In clear contrast the numerical exact MCF-calculation yields $\tau \simeq 1.3 \times 10^{-2}$ (for $\Gamma=20$ ), and $\tau \simeq 1.5 \times 10^{-2}$ (for $\Gamma=50$ ). Thus, although the current in this parameter regime is governed by Arrhenius-activated rates (the Arrhenius factor is of order 5 here) the regime of validity for the theory in [5] does not extend far enough to correctly predict the first current reversal. This finding is in accordance with previous comparisons for the Ornstein-Uhlenbeck limit in [7]. For $\Gamma=20$, the second reversal around $\tau=0.1$ happens to coincide accidentally with the theoretical predictions; note, however, that this current reversal crosses the $\tau$-axes with the wrong slope.

In Fig. 3 the crossover between the Ornstein-Uhlenbeck and the adiabatic limit is shown for $V(x)=V_{2}(x)$. For $\Gamma \rightarrow \infty$, the arrow indicates the current occurring in an Ornstein-Uhlenbeck noise driven ratchet with correlation time $\tau=1$. The opposite limit, $\Gamma \rightarrow 0$, can be treated within the adiabatic approximation, see Section 2.2. The mean current $J$ is evaluated by averaging the (analytically given) currents over an ensemble of Gaussian distributed, 


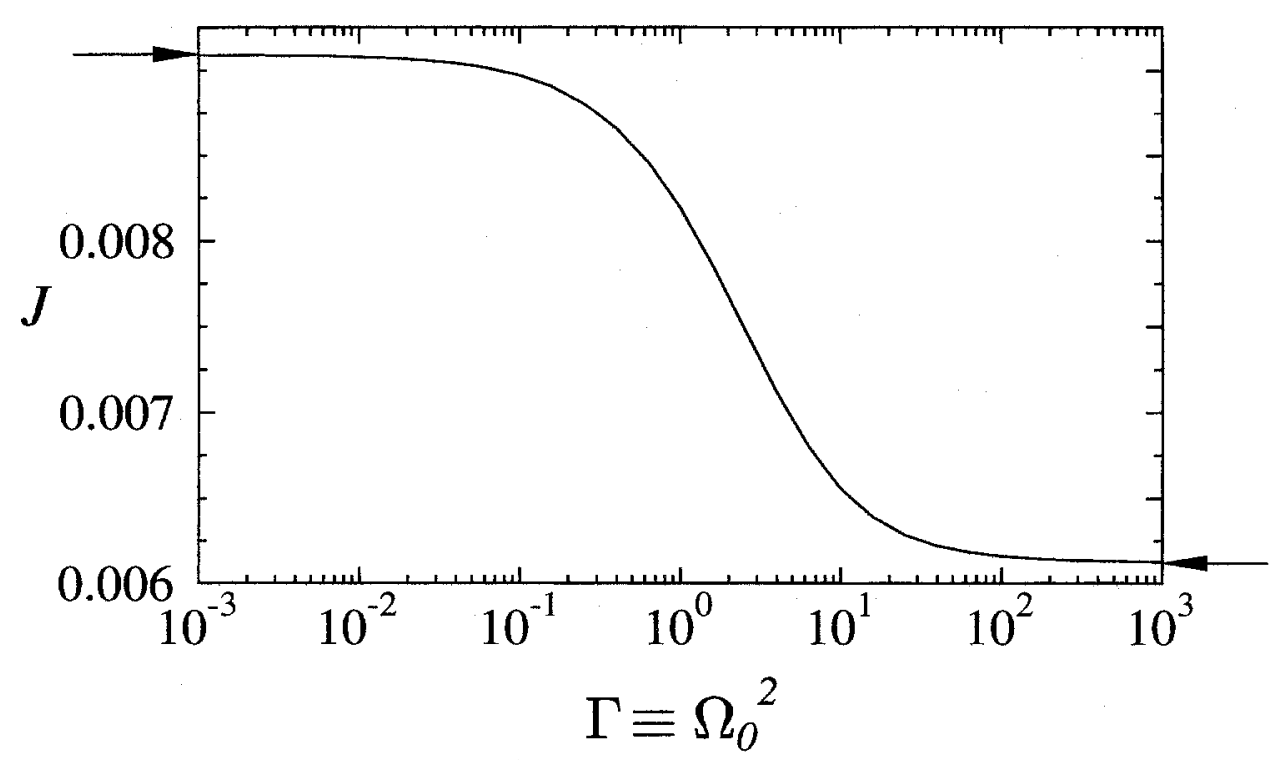

Fig. 3. The dependence of the probability current $J$ versus the damping parameter $\Gamma$ of the noise $\epsilon(t)$ is depicted for fixed $\tau=1$. The ratchet potential is $V(x)=V_{2}(x)$, and the noise intensities are $D=0.1$ and $Q=0.1$. The arrow at $\Gamma=10^{3}$ gives the current for Ornstein-Uhlenbeck noise with $\tau=1$, the arrow at $\Gamma=10^{-3}$ shows the adiabatic limit explained in the text. The characteristic crossover between these two limits occurs at $\Gamma=2 / \tau=2$.

statically biased potentials $U(x):=V(x)-x \epsilon[3,7]$. The result for this limit is depicted by an arrow. The theory in [5] again does not cover this parameter regime. It predicts a current reversal for $\Gamma=2$, which does not occur in the numerical calculation.

\section{Simulations and unified colored noise approximation}

For the potential $V(x)=V_{2}(x)$, we compare in Fig. 4 the results of MCF calculations (solid line) with direct simulations of the Langevin equations (1) and (2) (open circles), and with the 'unified colored noise approximation' (UCNA) (dotted) [14]. For the latter approximation, we substitute the processes $\epsilon(t)$ and $v(t)$ in (2) by suitably chosen auxiliary processes $q(t)=q(x(t), \epsilon(t))$ and $w(t)=w(x(t), \epsilon(t), u(t))$, yielding three new stochastic differential equations for $\dot{x}, \dot{q}$, and $\dot{w}$. For $\Gamma \gg 1$ and $\tau \ll 1$, the auxiliary processes $q(t)$ and $w(t)$ can be eliminated adiabatically. The resulting equation for $\dot{x}(t)$ reads [15]

$$
\dot{x}=\frac{1}{g(x)}\left[-V^{\prime}(x)+\sqrt{2 D} \xi_{1}(t)+\sqrt{2 Q} \xi_{2}(t)\right],
$$

where the prime (') denotes the derivative with respect to $x$, and

$$
g(x)=1-\frac{\partial}{\partial x} V^{\prime}(x)\left(\frac{1-\tau\left[\Gamma+V^{\prime \prime}(x)\right]}{\Gamma+V^{\prime \prime}(x)+(D / Q)\left\{\Gamma+\tau V^{\prime \prime}(x)\left[\Gamma+V^{\prime \prime}(x)\right]\right\}}\right) .
$$

This stochastic differential equation must be interpreted in the Stratonovich sense. Being one-dimensional, the approximative probability current $J_{\mathrm{UCNA}}$ can be obtained from (11) in closed form in terms of two quadratures $[1,15]$. This theoretical result is drawn as a dotted line in Fig. 4, the simulations and the MCF calculation for $\Gamma=20$ are given as open circles and as a solid line, respectively. The MCF result for Ornstein-Uhlenbeck noise (see in [7]) 


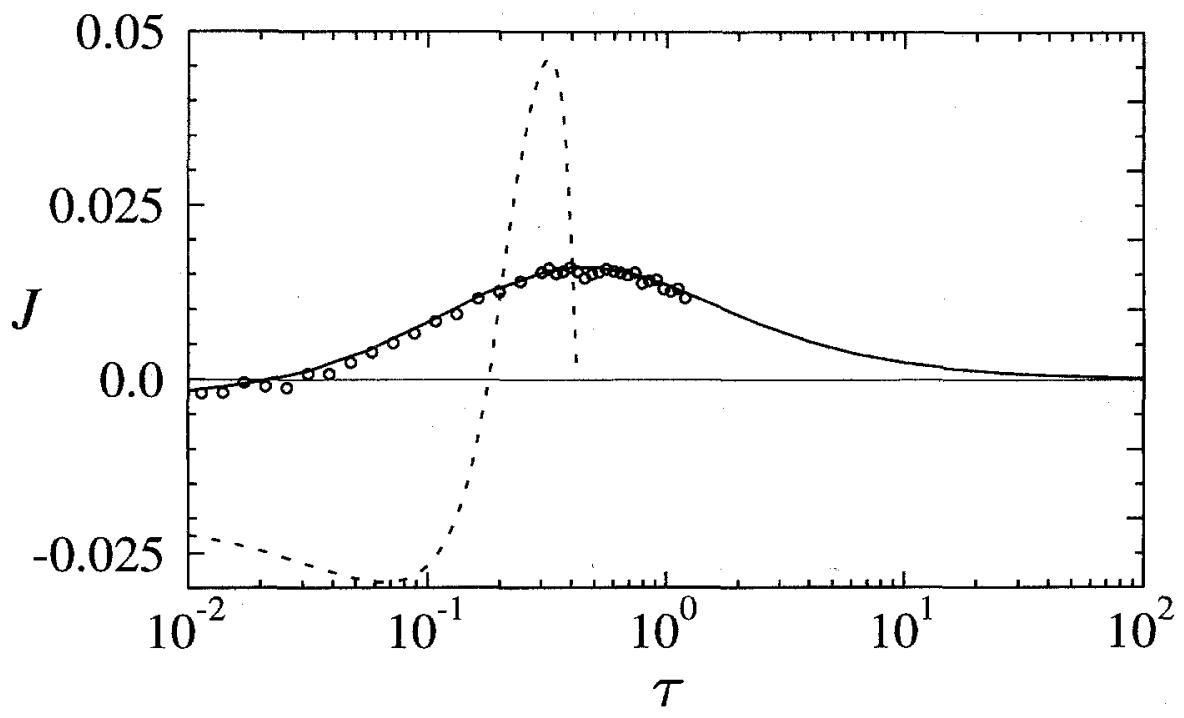

Fig. 4. The probability current $J$ is drawn versus the noise parameter $\tau$ for the ratchet potential $V(x)=V_{2}(x)$, being driven by white noise of intensity $D=0.1$, and harmonic noise with parameters $Q=0.25$ and $\Gamma=20$. Again, the current reversal occurs only when $\tau<2 / \Gamma$. The results were obtained from numerical MCF calculations (solid line) and from numerical simulations of the Langevin equations, which are depicted by open circles; the dotted line corresponds to the UCNA in Eq. (11).

does not exhibit a current reversal with increasing noise color $\tau$ when the ratchet potential $V(x)=V_{2}(x)$ is chosen. In contrast, the harmonic noise induced current possesses a current reversal at small $\tau$ which is only qualitatively predicted by the UCNA. This property of UCNA is remarkable because this approximation is a priori restricted in parameter space to very small $\tau$-values.

\section{Conclusion and outlook}

In this work we have presented precise numerical (MCF) calculations for the current arising in a ratchet system driven by white and harmonic noise $\epsilon(t)$. Several limits for harmonic noise were identified and corresponding results for the current were calculated. Simulations of the Langevin dynamics and the analytical (UCNA) predictions are found to be consistent with our precise MCF results. Our main findings is that Gaussian harmonic noise driven systems can possess multiple current reversals. This reversal of current occurs whenever the spectrum of harmonic noise is doubly peaked rather than decaying monotonically. The current reversals can be utilized for novel particle separation schemes if corresponding noise correlation times lie in disjunct parameter regimes, as indicated with Fig. 2. Hence, with this Brownian rectifier we encounter yet another case where the creative action of fluctuations - without the help of a Maxwell demon - supports an ordered state. A finite-valued directed particle current $\langle\dot{x}\rangle$, from which we can extract useful work.

\section{Acknowledgements}

This research has been supported by the Deutsche Forschungsgemeinschaft (RB and PH, Az. Ha1517/13-1). 


\section{Appendix A. Scaling of variables}

To show the correspondence between the 'real' parameters appearing in an experiment and those in the overdamped dimensionless equations (1) and (2), we start from the equations of motion in real time $\hat{t}$ for a particle of mass $m$ and friction coefficient $\gamma$ in a periodic potential $\hat{V}(\hat{x})=\hat{V}(\hat{x}+L)$ of period $L$, i.e.,

$$
m \frac{\mathrm{d}^{2} \hat{x}}{\mathrm{~d} \hat{t}^{2}}=-\frac{\partial \hat{V}(\hat{x})}{\partial \hat{x}}-\gamma m \frac{\mathrm{d} \hat{x}}{\mathrm{~d} \hat{t}}+\hat{\epsilon}(\hat{t})+\sqrt{2 m \gamma k_{\mathrm{B}} T} \xi_{1}(\hat{t}) .
$$

The absolute temperature is $T$ and $k_{\mathrm{B}}$ is the Boltzmann constant. Variables with hats denote quantities which have a dimension, like the real position of the particle $\hat{x}$ or the harmonic noise force $\hat{\epsilon}$. For the latter, the dynamics is given by

$$
\frac{\mathrm{d}^{2} \hat{\epsilon}}{\mathrm{d} \hat{t}^{2}} \equiv \frac{\mathrm{d} \hat{u}}{\mathrm{~d} \hat{t}}=-\hat{\Gamma} \hat{u}-\hat{\Omega}_{0}^{2} \hat{\epsilon}+\hat{\Omega}_{0}^{2} \sqrt{2 \hat{Q}} \xi_{2}(\hat{t})
$$

The Gaussian white noise sources $\xi_{1,2}(\hat{t})$ are $\delta$-correlated with respect to $\hat{t},\left\langle\xi_{i}(\hat{t}) \xi_{j}(0)\right\rangle=\delta_{i j} \delta(\hat{t}), i, j=1,2$. For the overdamped case $\gamma \rightarrow \infty$, we obtain the dimensionless equations (1), (2) from (A.1) and (A.2) by introducing the dimensionless variables $t=\gamma \hat{t}, x=\hat{x} / L, V(x)=\hat{V}(\hat{x}) / m \gamma^{2} L^{2}, D=k_{\mathrm{B}} T / m \gamma^{2} L^{2}, \epsilon=\hat{\epsilon} / m \gamma^{2} L, u=\hat{u} / m \gamma^{3} L$, $Q=\hat{Q} / m^{2} \gamma^{3} L^{2}, \Gamma=\hat{\Gamma} / \gamma$, and $\Omega_{0}=\hat{\Omega}_{0} / \gamma$. From these relations we immediately find $\tau=\gamma \hat{\Gamma} / \hat{\Omega}_{0}^{2}$. Hence it is evident that particles with a different friction coefficient $\gamma$ possess different effective correlation times $\tau$, and thus can be separated into different directions (see Figs. 2 and 4).

\section{References}

[1] P. Hänggi and R. Bartussek, in: Nonlinear Physics of Complex Systems - Current Status and Future Trends, eds. J. Parisi, S.C. Müller and W. Zimmermann (Springer, Berlin, 1996) p. 294; M. Kostur and J. Łuczka, Acta Physica Polonica B 27 (1996) 663.

[2] A. Ajdari and J. Prost, C. R. Acad. Sci. Paris 315 (1992) 1635; M.O. Magnasco, Phys. Rev. Lett. 71 (1993) 1477; J. Maddox, Nature 365 (1993) 203; 369 (1994) 181; 368 (1994) 287; I. Derényi and T. Vicsek, Phys. Rev. Lett. 75 (1995) 374; P. Reimann, R. Bartussek, R. Häußler and P. Hänggi, Phys. Lett. A 215 (1996) 26.

[3] R. Bartussek, P. Hänggi and J.G. Kissner, Europhys. Lett. 28 (1994) 459.

[4] C.R. Doering, W. Horsthemke and J. Riordan, Phys. Rev. Lett. 72 (1994) 2984; R.D. Astumian and M. Bier, Phys. Rev. Lett. 72 (1994) 1766; A. Mielke, Ann. Physik 4 (1995) 476; M. Bier and R.D. Astumian, Phys. Rev. Lett. 76 (1996) 4277; F. Marchesoni, Phys. Rev. Lett. 77 (1996) 2364.

[5] M.M. Millonas and M.I. Dykman, Phys. Lett. A 185 (1994) 65.

[6] J. Łuczka, R. Bartussek and P. Hänggi, Europhys. Lett. 31 (1995) 431; P. Hänggi, R. Bartussek, P. Talkner and J. Łuczka, Europhys. Lett. 35 (1996) 315; T. Hondou and Y. Sawada, Phys. Rev. Lett. 75 (1995) 3269; M.C. Mahato and A.M. Jayannavar, Phys. Lett. A 209 (1995) 21; J. Kula, T. Czernik and J. Łuczka, Phys. Lett. A 214 (1996) 14.

[7] R. Bartussek, P. Reimann and P. Hänggi, Phys. Rev. Lett. 76 (1996) 1166.

[8] P. Jung, J.G. Kissner and P. Hänggi, Phys. Rev. Lett. 76 (1996) 3436.

[9] R. Bartussek, in: Lectures on Stochastic Dynamics, eds. L. Schimansky-Geier and T. Pöschel (Springer, Berlin, 1997 ) p. 69.

[10] J. Rousselet, L. Salome, A. Ajdari and J. Prost, Nature 370 (1994) 446; S. Leibler, Nature 370 (1994) 412; T.D. Xie, P. Marszalek, Y.-D. Chen and T.Y. Tsong, Biophys. J. 67 (1994) 1247; L.P. Faucheux, L.S. Bourelieu, P.D. Kaplan and A.J. Libchaber, Phys. Rev. Lett. 74 (1995) 1504; R.D. Astumian and M. Bier, Biophys. J. 70 (1996) 637; I. Zapata, R. Bartussek, F. Sols and P. Hänggi, Phys. Rev. Lett. 77 (1996) 2292.

[11] L. Schimansky-Geier and C. Zülicke, Z. Phys. B 79 (1990) 451

[12] P. Jung and P. Hänggi, Phys. Rev. Lett. 65 (1990) 3365.

[13] M. Bier, Phys. Lett. A 211 (1996) 12; C. Berghaus, U. Kahlert and J. Schnakenberg, Phys. Lett. A 224 (1997) 243.

[14] P. Jung and P. Hänggi, Phys. Rev. A 35 (1987) 4464; A.J.R. Madureira, P. Hänggi, V. Buonamano and W.A. Rodriguez, Phys. Rev. E 51 (1995) 3849; R. Bartussek, A.J.R. Madureira and P. Hänggi, Phys. Rev. E 52 (1995) R2149.

[15] B. Lindner, Master's thesis, Humboldt Universität zu Berlin, Germany, 1996. 